\title{
Reflexión teórica de las estrategias flexibilizadoras en el marco de la globalización
}

\author{
Bonomie, María Elena* \\ Añez Hernández, Carmen**
}

\begin{abstract}
Resumen
Las estrategias flexibilizadoras por ser cónsonas con los cambios del entorno, comienzan a tener especificidad en las organizaciones para lograr la máxima eficiencia, competitividad, y estar en condiciones de dar respuestas a los lineamientos del mercado para poder posicionarse. Por lo tanto, deben aplicar estrategias en áreas específicas de la organización. Desde esta perspectiva, el trabajo tiene como propósito, realizar una reflexión teórica de las estrategias flexibilizadoras en el marco de la globalización. Para tal fin, se realizo una revisión bibliográfica, la cual permitió explorar los cambios significativos del término estrategia, ubicar las estrategias flexibilizadoras en el contexto de la globalización, así como analizar las ventajas y desventajas de las empresas al implementar dichas estrategias. Los resultados reflejan, que en el nuevo entorno globalizado las empresas establecen transformaciones gerenciales, como respuesta a los cambios e incertidumbres del entorno competitivo. Se concluye, que al implementar estrategias flexibilizadoras las empresas obtienen significativos resultados, entre los cuales se encuentra una mayor competitividad, a partir de la capacidad que puedan desarrollar para hacer frente a sus competidores. En este sentido, las empresas al tomar decisiones acertadas acerca de sus procesos productivos, los tipos de inversión, la tecnología a utilizar, entre otras, logran ventajas en los mercados servidos y captación de nuevos mercados.
\end{abstract}

Palabras clave: Globalización, empresas, estrategias flexibilizadoras, competitividad.

Recibido: 25-06-09. Aceptado: 21-10-09

* Economista, Magíster en Gerencia: Mención Industrial. Investigadora del Centro de Estudios de la Empresa de la Facultad de Ciencias Económicas y Sociales de la Universidad del Zulia. E-mail: mebonomie@yahoo.com

** Socióloga, Magíster En Intervención Social. Investigadora del Centro de Estudios de la Empresa de la Facultad de Ciencias Económicas y Sociales de la Universidad del Zulia.

E-mail: carmenanez@hotmail.com 


\title{
A Theoretical Reflection on Flexibilizing Strategies with the Framework of Globalization
}

\begin{abstract}
Because flexibilizing strategies are consonant with changes in the environment, they begin to have specificity in organizations in order to achieve maximum efficiency, competitiveness and be able to respond to market guidelines in order to position themselves. Therefore, strategies should be applied in specific areas of the organization. The purpose of this study is to reflect theoretically on flexibilizing strategies within the framework of globalization. A bibliographic review made it possible to explore significant changes in the term strategy, place flexibilizing strategies in the context of globalization, as well as analyze the advantages and disadvantages of implementing said strategies in the companies. Results reflect that in the new, globalized environment, companies establish managerial transformations as a response to changes and uncertainties in the competitive environment. Conclusions were that when companies implement flexibilizing strategies, they obtain significant results, among which are greater competitiveness based on the capacity they can develop to face their competitors. In this sense, when companies take correct decisions about their productive processes, types of investment, technology to be used, among others, they achieve advantages in the markets already served and in capturing new markets.
\end{abstract}

Key words: Globalization, companies, flexibilizing strategies, competitiveness.

\section{Introducción}

La globalización como proceso de la apertura de los mercados, el movimiento acelerado de bienes, servicios, y sobre todo, de las formas tangibles e intangibles de capital, contribuyen a reducir la distancia económica entre los países y los actores económicos, incrementándose de este modo la interdependencia económica. Sin embargo, la incertidumbre en los mercados es una constante, ante los cambios del entorno competitivo, lo cual ha generado en el sector empresarial distintas opciones estratégicas que permiten tomar decisiones cónsonas con el contexto globalizado para sobrevivir en el mercado. Dichas decisiones están enmarcadas en la necesidad de producir cambios y transformaciones en su interior, con el propósito de modernizar sus operaciones, procesos, estructuras, entre otros. Por ello, la aplicación de estrategias modernizadoras para operar de manera exitosa en el mercado, a fin de maximizar su ganancia, posesionarse en el mercado en las mejores condiciones con la mayor libertad posible para su crecimiento y movilidad.

En este sentido, las estrategias modernizadoras se orientan hacia las transformaciones gerenciales, surgiendo una serie de interrogantes en cuanto a ¿cómo adaptar las empresas a los cambios del mercado?, ¿Qué estrategias utilizar para simplificar las organizaciones y los procesos productivos? ¿Qué cambios introducir a los productos para competir?, ¿Cómo ampliar la competencia y el mercado?, ¿Cómo reducir los costos laborales?, ¿Qué estrategias utilizar para establecer relaciones con grandes y pequeñas em- 
Reflexión teórica de las estrategias flexibilizadoras en el marco de la globalización Bonomie, María Elena y Añez Hernández, Carmen

presas?, entre otras. Dichas interrogantes con sus respectivas acciones permiten a las empresas alcanzar la sobrevivencia y posicionamiento en los mercados nacionales e internacionales, de lo contrario se convertirán en recuerdo. Es por eso, que las empresas tienen que adaptarse a las exigencias del mercado y estar en continuo monitoreo de los posibles cambios para aminorarlos o neutralizarlos y seguir colocando sus productos en el mercado a pesar de sus exigencias.

Ante las condiciones generadas por la globalización, las empresas se esfuerzan para tener la capacidad de entender las grandes transformaciones inducidas por la difusión acelerada de las nuevas tecnologías, la interacción entre competidores como un sistema dinámico, así como hacer uso del conocimiento para predecir la disponibilidad de recursos que pueden dedicarse a diferentes usos y fines, la capacidad de predecir el riesgo y el rendimiento con suficiente exactitud y confianza, además de tener la disposición para actuar decididamente y comprometer esos recursos. Se trata, según Gamboa et al. (2001:141), "aprovechar las características de los diferentes mercados mundiales para colocar sus mercancías, producción o capital, donde maximicen su ganancia, en las mejores condiciones y con la mayor libertad posible".

Sin duda, las empresas ante este contexto, están definiendo e implementando estrategias enmarcadas en nuevos enfoques competitivos, técnicas y herramientas, que les permite mejorar constantemente la calidad de la producción, de los productos, de los servicios, en función de ampliar la competitividad y por supuesto, reducir costos en un mediano y largo plazo, convirtiéndose dicha tendencia en un desafío empresarial.

En este sentido, ante las condiciones de incertidumbre e inestabilidad de las empresas en los mercados, el trabajo pretende realizar una reflexión teórica acerca de las estrategias flexibilizadoras en el marco de la globalización. Para tal fin, se realizo una revisión bibliográfica, la cual permitió explorar los cambios significativos del término estrategia, ubicar las estrategias flexibilizadoras en el contexto de la globalización, así como analizar las ventajas y desventajas de las empresas al implementar dichas estrategias como respuesta a los cambios rápidos del entorno competitivo.

\section{Cambios significativos en el término estrategia}

Desde que surgió la dirección estratégica en la década del sesenta, hay consenso por los autores que abordan el tema, que los cambios bruscos que ocurren en el entorno es uno de los elementos que hicieron necesaria su aparición y posterior difusión por todo el mundo, hasta convertirse en una de las herramientas de dirección más empleadas con el avance de la competitividad en los mercados. El concepto de estrategia comienza a ser utilizado en el terreno de las operaciones guerreras, trasladándose su aplicación en época reciente a otras actividades humanas y, en particular a las actividades de negocios. Es por eso, que la estrategia es vista como una decisión presente con efecto futuro, convirtiéndose en un factor fundamental dentro de las organizaciones.

El término estrategia viene siendo tratado desde diferentes perspectivas e 
intereses, es por ello que su significado y aplicación está relacionado a un interés muy particular de las empresas.

En este sentido, realizando una revisión de los teóricos que han dedicado su esfuerzo al estudio del concepto estrategia como algo significativo en la acción, encontramos a Francés (2001:28), quien define la estrategia como un "plan de acción para alcanzar los objetivos en presencia de la incertidumbre." Mintzberg et al. (1996:50), abordan la estrategia también como un plan, pero la especifican como "el curso de acción conscientemente determinado, es decir, la guía o conjunto de guías, para enfrentar una situación, elaborada con antelación a las acciones a las cuales serán aplicadas y desarrolladas de manera consciente y con un propósito determinado". Para Marcuse (1997:151), la estrategia es "un plan de acción preparado para alcanzar un conjunto de metas preestablecidas; y pueden comprender un número de tácticas coordinadas e integradas para formar un todo coherente".

Es evidente, que los autores antes señalados, tienen su propia concepción de lo que es la estrategia y el propósito de la misma. Sin embargo, se puede observar que sus definiciones, están enfocadas sólo para que las empresas mejoren internamente, específicamente en la definición de objetivos, en la conformación de su organización y de su patrón de conducta, entre otros, sin tomar en cuenta la dinámica del entorno donde las empresas interactúan, el cual define las líneas de acción para su comportamiento. El termino estrategia se percibe como algo rígido y fuera de contexto al no tomar en cuenta los cambios, exigencias e incertidumbres del entorno. Es por eso, que la definición se torna limitante, ya que obvia como el mercado condiciona el comportamiento de las empresas, a través de los ciclos económicos, los cambios tecnológicos, preferencias de los consumidores, entre otros, que históricamente se han generado en el desarrollo del capitalismo.

Con la aparición de la concepción neoliberal, se plantea la necesidad de redimensionar el concepto de estrategia, con la finalidad de adaptarlo a las exigencias del mercado, de la competitividad, del posicionamiento de las empresas, en fin, con la concepción del nuevo desarrollo económico. Desde esta perspectiva, se orientan las nuevas visiones teóricas, en las cuales se vienen planteando como eje de la gerencia empresarial el contexto donde se desenvuelven. Por lo tanto, las estrategias comienzan a definirse como flexibilizadoras, ya que las empresas tienen que adaptarse y acompasarse a las directrices del mercado. Para Norman et al. (1998: 1), "la estrategia empresarial tiene que identificar las oportunidades en el mercado, el cual proporciona los marcos intelectuales, los modelos conceptuales y las ideas fundamentales que permite a los administradores de una compañía crear valor hacia los sujetos claves que interactúan con la organización, bien sean sus clientes o proveedores, creando a su vez una ganancia para los accionistas".

En consonancia con lo planteado, Díaz (1996:31), señala, que "hay una tendencia irreversible hacia la flexibilidad de los sistemas productivos y de las empresas, dada la creciente incertidumbre de los mercados de bienes, de capital y de trabajo; de los nuevos desafíos impuestos por la globalización y de las grandes transformaciones inducidas por la difu- 
Reflexión teórica de las estrategias flexibilizadoras en el marco de la globalización Bonomie, María Elena y Añez Hernández, Carmen

sión acelerada de nuevas tecnologías". De ahí pues, la necesidad de acuerdo a Thompson, et al. (1999), de implementar las estrategias con base a la conquista de una posición de negocios atractiva y desarrollar ventajas competitivas sustentables. Por lo tanto, las empresas deben desarrollar la capacidad de adaptarse a las condiciones cambiantes originadas por las acciones de los competidores, o por las modificaciones del medio ambiente económico en donde se desenvuelven, para poder alcanzar la expansión geográfica, la diversificación, el desarrollo de productos, entre otros, y no caer en el encogimiento, la desinversión, y la liquidación de empresas.

Desde esta perspectiva, la estrategia flexibilizadora presenta acciones más coherentes y unificadoras (entorno-empresa), lo cual permite tomar decisiones de acuerdo a las fortalezas y debilidades de la organización, tratando así de responder adecuadamente a las oportunidades y amenazas surgidas en el medio externo. Se trata por tanto, de buscar el camino mediante el cual una empresa define su negocio y vincularse con la economía contemporánea, donde el conocimiento del mercado, las relaciones, las competencias y los clientes, son los factores claves para mantenerse y posicionarse competitivamente.

En este orden de ideas, Fernández (2003: xii), plantea que, "las estrategias flexibilizadoras, constituyen un elemento central de la nueva forma de competir, en cualquiera de sus manifestaciones, operativa, estructural y estratégica", ya que estas permiten a la empresa adaptarse a situaciones cambiantes de forma evolutiva, y responder rápidamente a los cam- bios imprevistos, como por ejemplo: la aplicación de nuevas tecnologías, relaciones con proveedores, la subcontratación, entre otros.

Podríamos resumir a continuación, que las estrategias tienen una lógica, una razón de ser y un propósito bien definido, asunto que sintetiza Díaz (1996:2), en la siguiente idea, "las estrategias flexibilizadoras son sinónimo de ruptura en pos de un paradigma competitivo orientado sólo a favorecer la movilidad del capital". Se requiere por tanto, de reformas generales orientadas a la desregulación de los mercados y de políticas que permitan la flexibilidad de las empresas para competir más eficazmente.

Esto rompe con la concepción tradicional de estrategia, ya que la estrategia flexibilizadora esta orientada hacia la desverticalización de las empresas, frenando la rigidez de las organizaciones, de los procesos productivos y de las relaciones laborales. Visto de esta forma, se están implantando reformas que incentiven la flexibilidad de las empresas para competir en mejores condiciones y estar atentas a los cambios del mercado y de la economía como totalidad. De ahí la importancia de estar en condiciones para responder rápidamente como administrar los recursos, administración que debe basarse en conocimientos; en particular con las capacidades vinculadas a la flexibilidad, la innovación y la adaptación de carácter eminentemente dinámico.

\section{Globalización: Entorno de las estrategias flexibilizadoras}

La globalización impulsa la transformación acelerada de las economías nacionales en una sola economía mun- 
dial o global, esto obedece a que el neoliberalismo de un modo coherente con sus dogmas logra implantar el libre mercado tanto en los países desarrollados como subdesarrollados, suprimiendo la regulación de las intervenciones estatales para no tener interferencias de ningún tipo. De allí pues, que la globalización logra, de acuerdo a Montes et al. (2004:1), "el predominio del comercio libre; los intercambios de bienes y servicios muy intensos entre los países; en fin, una libertad plena de los movimientos de capital, apoyada en los avances de la informática y las comunicaciones".

De este modo, el neoliberalismo a través de la globalización logra, de acuerdo a Ponce (2001:1), que "las actividades económicas sean más eficientes y no encuentren trabas a su realización, lo que supone: liberalizar sin controles ni condiciones los flujos de capitales y el comercio; flexibilizar los mercados de trabajo y eliminar las cargas sociales de las empresas; en definitiva, que exista la desregularización de la vida económica y social".

En el mismo orden de ideas, Sotelo (1999:23), plantea que la globalización "es un proceso dinámico y un resultado histórico de la internacionalización del capital en función del proceso cíclico del capital dinero, productivo y mercancías". En este marco, las empresas comienzan a utilizar nuevos conceptos de organización, nuevas tecnologías sociales, es decir, un cambio cualitativamente nuevo, relacionado sobre todo, según Dombois y Preis (1993:11), "con las condiciones y las exigencias de los mercados y con los nuevos potenciales de tecnologías flexibles".

En efecto, el proceso globalizador conduce a que cambie "la manera de actuar de las organizaciones, generando una acelerada búsqueda de prácticas que permiten adecuar las operaciones a las nuevas exigencias del mercado, propiciando con ello un incremento de la competitividad" (Nava, 2004:34). Esta idea es compartida por Castells (1998:115), cuando señala que, "el proceso de globalización es retroalimentado con el incremento de la productividad; porque las empresas cuando se enfrentan a una fuerte competencia mundial, o luchan por ganar cuotas del mercado internacional, deben mejorar sus resultados".

Ante estas posturas, queda claro que en una economía globalizada, es difícil para las empresas alcanzar y mantener de una forma aislada elevados niveles de competitividad y enfrentar nuevas amenazas y oportunidades. Por lo tanto, se ven forzadas a administrar sus negocios con herramientas gerenciales modernas para intervenir en el mercado sin ningún tipo de restricciones e incluso crear las condiciones para su acceso.

En este sentido, las empresas utilizan estrategias flexibilizadoras con el fin de descentralizar la organización del trabajo, incorporando productos o servicios procedentes de otras empresas ubicadas en el mismo ámbito o foráneas, originando nuevas formas de producir y comercializar, lo cual fortalece el posicionamiento en los mercados nacionales e internacionales al establecerse redes de cooperación con sus posibles competidores. Al respecto, Rodríguez et al. (2002: 139), señalan que, "el proceso de globalización ha impuesto el paradigma del fortalecimiento de la competitividad, deslocalizando las actividades productivas para buscar una mayor productividad que 
Reflexión teórica de las estrategias flexibilizadoras en el marco de la globalización Bonomie, María Elena y Añez Hernández, Carmen

incida no sólo en la rentabilidad sino también configurando un nuevo orden social y político".

Sin duda, las empresas en coherencia con el entorno se están transformando en redes internas descentralizadas con cierto grado de autonomía para sus unidades productivas, tomando en cuenta las características de los países, de los mercados, de los procesos y de los productos. Esto implica la implementación de estrategias flexibilizadoras, entre ellas se encuentra la tercerización, la cual consiste en proveerse de un producto o servicio de un tercero, que según Añez et al. (2003:13), significa "la extracción de funciones con la finalidad de reducir costos y buscar una mayor flexibilidad $y$ adaptación a los constantes cambios del mercado, aplicando diferentes alianzas estratégicas". Por otra parte señalan, que la tercerización "implica la subcontratación de otras empresas para realizar las actividades o funciones que ya no son de interés para la organización, quedando esta concentrada en la gestión de su principal recurso, lo cual abre las puertas a una mayor expansión en el mercado" (Añez et al., 2003:13).

Esta estrategia, sin duda, se ha generalizando exitosamente en las empresas pasando progresivamente su aplicación hacia las actividades menos importantes y más costosas para la empresa, aportando un conjunto de ventajas desde el punto de vista técnico-productivo, que se traducen en mayor rentabilidad y menores costos. Con base a lo antes señalado, Añez et al. (2003: 12) plantean que, "el establecimiento de empresas globales conduce a sustituir las estructuras organizacionales jerárquicas, verticales, con especialización del trabajo y diseñadas para producir en masa; por una organización plana que permite flexibilizar todos los aspectos organizacionales, con la finalidad de adaptarse a las fluctuaciones y exigencias del mercado nacional y global". De este modo, se genera una nueva división del trabajo, con un mayor número de empresas especializadas, ligadas a través de una densa red de relaciones interempresariales y entre compradores y suministradores. Avanzar en esta dirección permite a las empresas integrar el proceso de modernización al posicionamiento en el mercado internacional.

El avance vertiginoso que ha tenido la globalización en los últimos años, ha obligado a las empresas a asumir otras estrategias flexibilizadoras, Peixoto (1998:11) al respecto, señala que, "acuerdos y asociaciones entre empresas están siendo presentados, como la superación de formas tradicionales y antiguas de organización de la producción e indicadas como estrategias empresariales más eficaces en ambientes económicos de incertidumbre". Por otra parte, a través de estas estrategias se logra enfrentar y redefinir las actividades, procesos, comercialización, entre otros, conforme a los cambios rápidos del entorno.

Por consiguiente, las empresas se encuentran en un proceso de cambio, que conlleva hacia un carácter sistémico de innovación en los procesos de las organizaciones, específicamente en el proceso productivo y de comercialización. En concordancia a lo señalado, Añez et al. (2003:12), señalan, que las empresas se están conformando en "alianzas estratégicas, fusiones, adquisiciones, entre otras, para poder sobrevivir a los cons- 
tantes cambios y a las pautas que establece el mercado", debido a que el capital por sí sólo no puede producir ni competir en el mercado, y mucho menos acceder a nuevos espacios geográficos.

A nivel mundial se ha demostrado que las empresas al asociarse se enfrentan en mejores condiciones para competir en un mercado global. Es por ello, que Trigo et al. (2000:9), plantean que "las alianzas estratégicas son acuerdos formales de colaboración entre empresas o entre empresas y organizaciones de otra índole que reúnen tres características a) están relacionadas con su actividad productiva, b) son tendientes a alterar el posicionamiento en el mercado o abrir mercados; c) en ellas la autoridad y la toma de decisiones se comparten, con lo que se adoptan las resoluciones sin recurrir a métodos jerárquicos de coordinación". Las alianzas estratégicas en definitiva permiten que las empresas mejoren su gestión, compartan riesgos, tecnología, información y complementen las capacidades de la asociación para desarrollar productos de calidad y diversificados.

Por otra parte, dentro de las estrategias flexibilizadoras se encuentran las fusiones y adquisiciones. Según Mascareñas (1996:1)," las fusiones son acuerdos de dos o más sociedades, jurídicamente independientes, por el que se comprometen a juntar sus patrimonios y formar una nueva sociedad". Es decir, las fusiones son estrategias en la que la empresa decide unirse para formar una nueva; en este tipo de estrategia, al igual que en las alianzas, la sinergia juega un papel preponderante, al unir capacidades, esfuerzos y ventajas de dos firmas. Dicha estrategia permite a las empresas adap- tarse a los cambios rápidos del entorno y alcanzar los objetivos organizativos, superando las nuevas coyunturas de las economías y del mercado competitivo.

En cuanto a la estrategia de adquisición, se implementa cuando una empresa o grupo de empresas compra parte de otra, adquiriendo cierto poder sobre la misma. Cuando una empresa o grupo de empresas toma posesión de otra de menor tamaño, obteniendo el poder total de la misma, se entiende esta adquisición total como una absorción. Al respecto, Nava (2004:49), sostiene, que dichas estrategias "han dado origen a una nueva realidad, en la cual la concentración del capital marca la pauta".

Ahora bien, la globalización además de imponer que las empresas establezcan relaciones de alianzas, fusiones, o asociaciones, entre otras, ha derivado la importancia de modernizar e introducir estrategias tecnológicas. Estas estrategias de acuerdo con Mendizábal (2002:1), implica "nuevas tecnologías, conformando una sociedad del conocimiento que permite desarrollar la intercomunicación entre las diferentes empresas y entre los diversos departamentos en el interior de cada empresa". En efecto, la introducción de nuevas tecnologías transforman los sistemas de gestión y los procesos mismos, lo cual conduce simultáneamente ha incorporar cambios significativos en la organización del trabajo, configurándose una nueva gestión estructurada en base a los grupos de trabajo.

En este sentido, las nuevas tecnologías permiten modificar la estructura de los procesos de trabajo, cambiando sus tiempos en breves períodos y diversificando la producción. Es por esto, que 
Reflexión teórica de las estrategias flexibilizadoras en el marco de la globalización Bonomie, María Elena y Añez Hernández, Carmen

existe la tendencia en las empresas de incrementar la flexibilidad en los talleres de fabricación a objeto de poder diversificar mejor los productos, ya que el antiguo modelo basado en grandes fabricas para la producción en masas, se sustituye por talleres flexibles fabricando en series cortas, con mandos electrónicos que permiten programar cambios rápidos en la gama de productos y en los tiempos de producción.

La globalización a su vez, conduce a las compañías a modernizar los sistemas de información, con el propósito de estar comunicados con sus filiales, proveedores, distribuidores, entre otros sujetos. El ritmo del mercado ha sustituido las viejas formas de comunicación por la informática y grandes ordenadores en red, con lo cual puede obtenerse información importante relacionada con cualquier aspecto en tiempo real. Esta idea se concretiza en el planteamiento de Meleán (2004:75), cuando señala, que la modernización en informática como estrategia, "requiere de cambios en los paradigmas y en la cultura organizacional de la empresa, puesto que ofrece nuevas modalidades de comunicación entre distintos lugares de la geografía mundial"; la resistencia por parte de los miembros de la organización al uso de esta herramienta implica quedar fuera de la información real y precisa de los acontecimientos del mercado, lo cual incide en la toma de decisiones. Al respecto, Gamboa et al. (2003: 597), establecen que "los desarrollos informáticos y en telecomunicaciones exigen a las empresas usar creativamente los sistemas de información y de gestión: para centralizar la toma de decisiones porque potencia enormemente la posibili- dad de coordinación y control a lo largo y ancho de las diferentes estructuras organizativas".

Sotelo (1999:24), introduce a la modernización de los sistemas de información otro propósito, como es elevar la productividad del trabajo y valorar el capital, esta idea la sustenta en los siguientes términos: "la tecnología informática, en tanto capital productivo que produce mercancías, servicios y productos diferenciados, interactúa en el proceso de valorización del capital y constituye un instrumento eficaz para elevar la productividad del trabajo".

Relacionado a las anteriores estrategias, para que una empresa logre competitividad y adaptación a las líneas del mercado, tiene que introducir la innovación, definida esta por Mendizábal (2002:7), como "una estrategia competitiva, la cual se presenta de dos formas, la primera consiste en la adquisición de innovación existente fuera de la empresa y que afecta el proceso implementado por esta, y la segunda tiene que ver con la aplicación de la innovación, operacionalizándose a través de cambios organizacionales".

En cuanto a la primera forma de innovación, ésta surge por un adelanto tecnológico y/o organizativo realizado en el exterior y que afecta al proceso productivo de la empresa. Ello, exige la conexión con el entorno ecológico y tecnológico empresarial con el objeto de acceder a la dinámica exterior en cuanto a nuevas maquinarias y nuevas patentes. Con respecto a la segunda forma de innovación, según Mendizábal (2002:8), "tiene que ver no tanto con la innovación en sí, sino con su aplicación, es decir a los cambios organizacionales tales como, la mejora 
continua, que no más que la suma de mejoras pequeñas que muchas veces no son apreciables, pero que inciden en el logro de saltos cualitativos en el proceso, en cambios sustanciales en las instalaciones, y en las trasformaciones requeridas para hacer las cosas.

En la perspectiva de la globalización las estrategias flexibilizadoras no solo se aplican a la organización y a los procesos, sino que abarca también a la fuerza de trabajo. En este sentido, Sotelo (1999:117), plantea que, "el predominio y la dinámica del patrón capitalista neoliberal, demandan transformaciones impostergables en los sistemas productivos, los procesos de trabajo y las legislaciones laborales". Esto conlleva a que se institucionalice en las empresas la estrategia de flexibilización laboral, la cual se caracteriza por un profundo proceso de desregulación de las condiciones laborales de los trabajadores, perfilándose mercados laborales: segmentados, polivalentes, precarizados y ajustados a los imperativos que van definiendo la productividad y la competitividad.

La flexibilidad laboral se convierte en una estrategia que cumple dos propósitos: la primera, relacionada con la adaptación de los mercados de trabajo a las innovaciones tecnológicas y a los cambiantes ritmos del ciclo económico, y la segunda, es hacer más competitivas a las empresas, con el aumento de la intensidad del trabajo, la disminución de los salarios y la desregularización de la protección de los trabajadores.

En concordancia con lo señalado, es evidente que el impacto de la globalización en las empresas, no puede considerarse simplemente como un proceso de ampliación de mercados, sino también implica un proceso de transformaciones y cambios en su interior, lo cual se ha convertido en un reto para modernizar sus operaciones y adaptarse a los cambios, por ello la necesidad de aplicar estrategias proactivas que les permita operar de manera exitosa.

\section{Ventajas y desventajas de las estrategias flexibilizadoras}

En la actualidad, el entorno cambiante hace que las empresas diseñen estrategias flexibilizadoras para alcanzar la competitividad, expandir dimensiones del mercado, desarrollar tecnología e innovación, reducir costos laborales, entre otros, es decir, las empresas consideran a las estrategias como una prioridad competitiva para mejorar su posición ante un proceso de cambio que se despliega aceleradamente. Sin embargo, es importante analizar con detenimiento las implicaciones que pueden generar la aplicación de estas estrategias.

Las empresas ante la agresiva competitividad y los altos costos no pueden gerenciar aisladamente, por lo tanto establecen relaciones interempresariales. En consecuencia, se agrupan y reagrupan de diferentes maneras para fortalecerse y compartir responsabilidades, logrando así enfrentar los cambios del entorno, reducir costos de operaciones e inversiones, aplanar sus estructuras y posicionarse en el mercado.

Generalmente las grandes empresas establecen convenios con medianas y pequeñas empresas, lo cual las beneficia al desverticalizar sus funciones y operaciones. Con esta relación, las empre- 
Reflexión teórica de las estrategias flexibilizadoras en el marco de la globalización Bonomie, María Elena y Añez Hernández, Carmen

sas grandes reducen costos, contratan personal capacitado, tecnología e información, ceden a terceros parte de los procesos productivos, dedicándose a funciones que son clave para la empresa. Con dicho reordenamiento se establece una relación de interdependencia entre las empresas, interviniendo simultáneamente un conjunto de agentes y actividades económicas para alcanzar el éxito de la alianza.

Sin embargo, se debe considerar que al establecerse los convenios, las grandes empresas mantienen sus capacidades de gestión, autonomía e independencia, llevando a cabo el control de la actividad que es objeto del acuerdo, y más aun a pesar de la interdependencia de las empresas el éxito en la competitividad es exclusiva de la empresa que contrata, lo cual es extensivo al resto de sus actividades.

En lo esencial, las grandes empresas buscan seguridad, información, un mayor grado de especialización, de acuerdo con los requerimientos del mercado, combinando competencias, utilizando know-how de otras empresas, dividiendo los riesgos y costes para explotar nuevas oportunidades.

Es necesario resaltar que las relaciones con las pequeñas empresas pasa por un criterio de selección establecido por la gran empresa, tal es el caso de la mano de obra, la cual debe ser altamente calificada. Dicha exigencia obedece a la necesidad de extraer a bajos costos: experiencia, conocimiento tácito, códigos de comunicación y procedimientos, los cuales son utilizados e integrados en el desarrollo de las innovaciones.
Sin embargo, conviene señalar que las estrategias flexibilizadoras "son una forma de organización que presenta un equilibrio inestable, debido a los diferentes intereses de las organizaciones dentro de la red" (Vázquez, 2005:52), situación que afecta tanto a las grandes empresas, como a las PYMES al no poderse adaptar estas últimas según Añez (2007) al entorno dinámico, ocasionando una alta mortalidad de dichas unidades de producción, así como la ruptura e inestabilidad de la cadena. El riesgo de las estrategias flexibilizadoras es la no permanencia en el tiempo ni en la organización, ya que las exigencias del mercado son cambiantes, siendo por lo tanto sustituidas las estrategias por otras cuando se considera que han dejado de ser útiles.

Evidentemente las empresas grandes también sufren las incertidumbres del mercado. Al introducirse cambios como aumento de aranceles, apertura del capital extranjero y las importaciones, entre otros, estas se enfrentan a una serie de conflictos que conducen a tomar decisiones como disminuir sus relaciones con los proveedores y a redimensionar los procesos productivos, rompiéndose la implementación de las estrategias flexibilizadoras (Añez, 2007).

Con respecto a las estrategias laborales, el contrato de trabajo más utilizado es el eventual o determinado, el cual se configura para satisfacer resultados concretos. La relación laboral, por su propia naturaleza, está destinada a agotarse en el tiempo. A pesar que las empresas recurren a este tipo de contrato de trabajo para reducir costos laborales, esta estrategia tienen desventajas para las empre- 
sas, ya que el trabajador no se identifica con la filosofía empresarial, al cesar el contrato de trabajo el conocimiento y experiencia obtenido por la fuerza laboral es trasladado a otras empresas, por lo tanto la información de las empresas deja de ser secreto y aprovechada por la competencia. El conocimiento ajeno representa la habilidad de acceder de manera flexible a las tecnologías modernas de las empresas, convirtiéndose en una fuente de ventaja competitiva, al llevarse los conocimientos adquiridos.

En cuanto a los adelantos científico-técnicos y administrativos, las empresas para accesar a dichos adelantos, han dado paso a una organización que, a fin de adaptarse al entorno, tiende a la desburocratización y a la búsqueda de respuestas rápidas al mercado, configurando relaciones de su estructura en función del elemento tecnológico e información, valorando el conocimiento como verdadera fuente de valor. Sin embargo de acuerdo a Guerra y Guerra (2004), la corporación actual está estructurada alrededor de capas de administración. Muchas de estas capas son redes de información, y como cualquier red son muy débiles. Tanto lo tecnológico como los sistemas de información deben ser muy inteligentes, ya que ambos se vuelven obsoletos a un ritmo increíblemente rápido.

\section{Conclusiones}

Los cambios aportados por la globalización han derivado una redefinición del concepto tradicional de estrategia, adaptándolo a las nuevas formas de pro- ducción, y a los cambios del entorno. La llegada de la globalización acarrea cambios evidentes en las estrategias empresariales, pasando de una búsqueda de la diferenciación como modelo de ventaja competitiva frente a los competidores, a estrategias flexibilizadoras que respondan con adecuada certeza a la alta incertidumbre y turbulencia del contexto, con el fin de crear un espacio en el mercado o al menos sobrevivir en este gran escenario global.

Las estrategias flexibilizadoras se convierten en un desafío para las empresas, ya que con ellas pueden afrontar y adoptar nuevos enfoques organizacionales, competitivos, así como técnicas y herramientas que coadyuven a mejorar constantemente la calidad de la producción, de los productos y de los servicios que presta la organización.

Por lo tanto, las empresas al implementar estrategias flexibilizadoras obtienen significativos resultados, ya que desarrollan capacidades que permiten tomar decisiones acertadas para enfrentar a sus competidores en los mercados. Para su logro es indispensable construir un modelo coherente que establezca acciones, prioridades, metas y objetivos, adecuados a las oportunidades, amenazas del entorno, así como a las debilidades y fortalezas de la organización. Por otra parte, la utilización optima de estrategias flexibilizadoras aplicadas a los recursos humanos, implica para las empresas la reducción de costos de producción, ya que estos representan la clave para poder expandirse y ofrecer precios competitivos en el mercado. 
Reflexión teórica de las estrategias flexibilizadoras en el marco de la globalización Bonomie, María Elena y Añez Hernández, Carmen

\section{Referencias Bibliográficas}

Añez, Carmen; Boscán, Roberto; Useche, María C. (2003) "Estrategias Globalizadoras. Tendencia Histórica del Capitalismo". Revista Economía No. 16. Universidad de los Andes. Mérida -Venezuela.

Añez, Carmen; Useche, María C. (2003). "Flexibilización de las relaciones de trabajo y su incidencia en la fuerza laboral". Revista FACES. Universidad de Carabobo. Facultad de Ciencias Económicas y Sociales. Año 12. Número 23. Enero junio.

Añez Hernández, Carmen (2007) "Pequeñas Empresas en las Cadenas Productivas: Crecimiento o Dependencia". Revista Análisis de Coyuntura Vol. XIII, No 1. Enero-Junio 2007. Universidad Central de Venezuela.

Castells, Manuel (1998) La Era de la Información. Economía, Sociedad y Cultura. Vol. 1. La Sociedad Red. Alianza Editorial. Madrid. España.

Díaz, Alvaro (1996) "Las Dimensiones Macroeconómicas y Empresariales de la Flexibilidad Productiva". Revista Capítulos SELA. Edición No. 48. Industria, tecnología y competitividad. Octubre - diciembre 1996. Pag. 9. Www.lanic.utexas.edu/sela/AA2K/ ES/cap/N48/rcap486.htm consulta. 17-03-2004

Díaz, Álvaro (1996). "Flexibilidad Productiva en las Economías de la Región y nuevos Modelos de Empresa". Boletín Cinterfor. No. 137., Octubre Diciembre.

Dombois, Rainer y Pries, Ludger (1993). Modernización Empresarial. Tendencias en América Latina y Europa. Fundación FRIEDDRICH Ebert de Colombia (FESCOL). Editorial Nueva Sociedad. Colombia.

Fernández Esteban, Avella Lucía; Fernández, Martha (2003). Estrategias de Pro- ducción. Mc Graw Hill. Pag. 9-169170. México.

Francés, Antonio (2001). Estrategia para la Empresa en América Latina. Ediciones Caracas, Venezuela.

Guerra, Alexei y Guerra, Alexis (2004). EI nuevo paradigma organizativo en el escenario global y posmoderno. Caracas-Venezuela.

Gamboa Cáceres, Teresa; Arellano Rodríguez, Madelein; Nava Vásquez, Yuneska (2001). "Estrategias Empresariales: una aproximación a una topología". Revista Venezolana de Ciencias Sociales. UNERMB, Vol. 5 No. 2. Universidad Nacional Experimental Rafael María Baralt. Págs. 132-154. Maracaibo-Venezuela.

Gamboa Cáceres, Teresa; Arellano Rodríguez, Madelein; Nava Vásquez, Yuneska (2003). "Estrategias de Modernización Empresarial: Procesos, Productos y Fuerza de Trabajo". Revista Venezolana de Gerencia. Año 8 No. 24. Universidad del Zulia. Págs. 592-606. Maracaibo Venezuela.

Gamboa Cáceres, Teresa; Arellano Rodríguez, Madelein; Nava Vásquez, Yuneska (2003). "Actores y Fines de las Estrategias Empresariales. Una Reflexión desde las Pequeñas y Medianas Empresas". Revista Visión Gerencial. Centro de Investigaciones de Desarrollo Empresarial (CIDE). Año2. No. 1. Vol. I. Enero - junio 2003. Universidad de los Andes. Páginas 2839. Mérida, Venezuela.

Marcuse, Robert (1997). Diccionario de Terminología Financiera. Ediciones Manantial. Buenos Aires-Argentina. $N^{\circ}$ de Páginas 317.

Mascareñas Pérez-Iñigo, Juan (1996). Manual de Fusiones y Adquisiciones de Empresas. Segunda Edición. McGraw.Hill. España. № de Págs. 465 
Meleán, Rosana (2004). Estrategias para Gerenciar Costos de Producción en Industrias de Pastas Alimenticias del Estado Zulia - Venezuela, Trabajo de Grado. Maestría Gerencia de Empresas. Mención Gerencia Industrial. Universidad del Zulia. Maracaibo - Venezuela.

Mendizábal, Antxon (2002). Globalización y Gestión del Conocimiento en la empresa moderna. Http://monedahn.terra.com/moneda/noticias/mnd4861. htm . Consultada el 04/09/2004.

Montes, Pedro y Toledano, Diosdado (2004). Globalización $=$ Capitalismo + Neoliberalismo. Http://www.espaimarx. org/2_12.htm. Fecha de consulta 1301-2005

Nava Vásquez, Yuneska (2004). Fusiones y Adquisiciones como Estrategias Empresariales Bancarias. Trabajo de Grado. Maestría Gerencia de Empresas. Mención Gerencia Financiera. Universidad del Zulia. Maracaibo Venezuela.

Norman, Richard y Ramírez, Rafael (1998). De la Cadena de Valor a la Constelación de Valor Diseñando una Estrategia Interactiva. Http://mailweb. udlap.mx/ jduran/articulos/IKEA.htm Fecha de consulta 14-02-05.

Peixoto de Alburquerque, Paulo (1998). "Las estrategias empresariales y el nuevo ambiente económico. La realidad virtual del cambio". Revista Venezolana de Gerencia, Año 3 No. 5. Pág. 9-24. Universidad del Zulia. Maracaibo- Venezuela.

Ponce, Pedro (2001). La globalización. www.mural.uv.es/juasa.jua/laglobalizacion.htm. Fecha de consulta 2404-2004

Rodríguez Medina, Guillermo; Balestrini, Solange; Balestrini, Sara; Meleán, Rosana; Rodríguez, Belkis (2002). "Análisis Estratégico del Proceso Productivo en el Sector Industrial". Revista de Ciencias Sociales. Vol. VIII. No. 1 Enero-Abril. Páginas 135-156. Universidad del Zulia. Maracaibo-Venezuela.

Sotelo Valencia, Adrián (1999). Globalización y Precariedad del Trabajo en México. Ediciones el caballito. México.

Trigo, Joaquín y Drubis, Antoni (2000). Alianzas Estratégicas. Las Claves y la Práctica de la Cooperación entre Empresas. Ediciones Gestión 2000. Barcelona. España.

Thompson, Arthur A. y Strickland III, A.J. (1999). Administración Estratégica. Conceptos y Casos. Undécima Edición. Mc. Graw Hill. México.

Vázquez Barquero, Antonio (2005). Las nuevas Fuerzas del Desarrollo. Antoni Bosch editor. Barcelona España. 